\title{
Intermediate Dynamo Models
}

\author{
Gary A. Glatzmaier ${ }^{1}$ and Paul H. Roberts ${ }^{2}$ \\ ${ }^{1}$ Earth and Environmental Sciences Division, Los Alamos National Laboratory, \\ Los Alamos, NM 87545, U.S.A. \\ ${ }^{2}$ Institute of Geophysics and Planetary Physics, University of California, Los Angeles, CA 90024, U.S.A.
}

(Received November 30, 1992; Revised April 6, 1993; Accepted April 12, 1993)

\begin{abstract}
We describe a nonlinear, axisymmetric, spherical-shell model of a planetary dynamo. This intermediate-type dynamo model requires a prescribed helicity field (the alpha effect) and a prescribed buoyancy force or thermal wind (the omega effect) and solves for the axisymmetric time-dependent magnetic and velocity fields. Three very different time dependent solutions are obtained from different prescribed sets of alpha and omega fields.
\end{abstract}

\section{Introduction}

Nearly all numerical models of planetary dynamos during the last thirty years have been "mean field" kinematic models which require prescribed time-independent axisymmetric (alpha and omega) effects of some hypothetical three-dimensional convection and solve for the evolution of only the two-dimensional axisymmetric magnetic fields (ROBERTS and SOWARD, 1992). Kinematic models have been used because of their simplicity and relatively small computing requirements. They have been very successful for studying the "weak field" regime, in which the feedback of the magnetic Lorentz force on the dynamics is small and can be treated as a small perturbation to the dynamics.

However, planetary dynamos, like those in the metallic liquid interiors of Jupiter and the Earth, are probably operating in the "strong field" regime for which the Lorentz force is as strong as the Coriolis force and therefore cannot be treated as a perturbation. In an attempt to model this "strong field" regime without resorting to a self-consistent three-dimensional model, nonlinear intermediate dynamo models have been developed that assume the Lorentz force is comparable with the Coriolis force (BRAGINSKY, 1975, 1978; BRAGINSKY and RoBERTS, 1987; ROBERTS, 1989; BARENGHI and JONES, 1991). They require a prescribed axisymmetric helicity field (the alpha effect) and a prescribed axisymmetric thermal wind or buoyancy force (the omega effect). However, in addition to solving for the axisymmetric magnetic field, they solve for the axisymmetric meridional circulation, magnetic wind, and geostrophic wind that are generated by the Lorentz force. Most intermediate models make the inviscid magnetostrophic approximation, which neglects the inertial and viscous terms, and they assume a viscous Ekman boundary layer that determines the geostrophic wind by balancing the $z$-independent magnetic torque. Another approach has been used (OLSON, 1989) that also prescribes the alpha effect and makes the magnetostrophic approximation but solves a prognostic heat equation to provide a buoyancy force and uses a linear viscous drag throughout the fluid shell to balance the $z$-independent magnetic torque in order to solve for the geostrophic wind. All these intermediate models obtain axisymmetric solutions by solving an initial value problem.

The full three dimensional magnetohydrodynamic dynamo problem is quite formidable (Gilman and Miller, 1981; Gilman, 1983; Glatzmaier, 1984, 1985a, b; Fearn and Proctor, 1987; ZHANG and Busse, 1989), especially if one wishes to study the highly supercritical, strong magnetic field, rapidly rotating, low viscosity regime. It is necessary to follow, with high spatial resolution, the evolution of the vector magnetic field, the vector fluid velocity, the entropy, pres- 
sure and chemical composition, all of which are three dimensional and each of which provides nonlinear feedbacks on the others. It is this complexity that precludes any "back of the envelope" intuition about the actual structure and evolution of the planetary magnetic fields.

In this paper, we present another intermediate type dynamo model and describe the axisymmetric solutions obtained from three different sets of prescribed alpha and omega fields. We also describe two different versions of the model: one neglects the inertial terms and prescribes a thermal wind and the other retains the inertial terms and prescribes a buoyancy force. Our model differs from other intermediate dynamo models by not making the inviscid magnetostrophic approximation. That is, we prescribe a viscous diffusivity throughout the fluid that is comparable with the magnetic diffusivity. We therefore resolve the Ekman boundary layers and the internal Stewartson layer on the imaginary cylinder tangent to the inner core (HoLlERBACH, 1992). This choice of using a Prandtl number of order unity in the bulk of the fluid is intermediate between the zero Prandtl number used in inviscid magnetostrophic models and the infinite Prandtl number used by ZHANG and Busse (1990). However, in our solutions, the work per volume done by the viscous forces is several orders of magnitude greater in the Ekman boundary layers than it is in the bulk of the fluid shell.

\section{The Model}

Our model is based on the axisymmetric magnetohydrodynamic equations for fluid motion and magnetic field generation in a rotating spherical shell with a prescribed alpha effect that parametrizes helical fluid motion and a prescribed omega effect that represents either a buoyancy force or a thermal wind. We assume a Boussinesq fluid with constant reference state density, $\rho_{0}$, and constant viscous and magnetic diffusivities, $\nu_{0}$ and $\eta_{0}$; and we neglect Reynolds stress. The perturbation equations for an electrically-conducting fluid in a spherical shell of outer and inner radii, $r_{0}$ and $r_{i}$, rotating with angular velocity $\Omega$, with an inward directed gravitational field $g_{0}\left(r / r_{0}\right)$ are

$$
\begin{aligned}
& \nabla \cdot \mathbf{v}=0 \\
& \frac{\partial \mathbf{v}}{\partial t}=-\frac{1}{\rho_{0}} \nabla p-g_{0}\left(\frac{r}{r_{0}}\right)\left(\frac{\rho}{\rho_{0}}\right) \hat{\mathbf{r}}+2 \mathbf{v} \times \Omega+\frac{1}{4 \pi \rho_{0}}(\nabla \times \mathbf{B}) \times \mathbf{B}+\nu_{0} \nabla^{2} \mathbf{v}, \\
& \nabla \cdot \mathbf{B}=0 \\
& \frac{\partial \mathbf{B}}{\partial t}=\nabla \times(\mathbf{v} \times \mathbf{B}+\alpha \mathbf{B})+\eta_{0} \nabla^{2} \mathbf{B},
\end{aligned}
$$

where $\mathbf{v}$ is velocity, $\mathbf{B}$ is magnetic field, and $p$ and $\rho$ are the pressure and density perturbations. We use electromagnetic units with magnetic permeability set to $4 \pi$. The function $\alpha$ is the prescribed alpha field and, for the solutions presented here, it multiplies only the longitudinal (toroidal) part of $\mathbf{B}$ and this product is added only to the meridional (poloidal) part of $\mathbf{B}$. The normalized density perturbation in the buoyancy term is either prescribed or, if a thermal wind is prescribed, it is set to zero.

Our solutions are not constrained to be either symmetric or anti-symmetric with respect to the equator. The inner and outer boundaries are impermeable and non-slip, except for the spherically-symmetric part of the angular velocity which has a stress free inner boundary condition to allow for solid body rotation of the inner core. The boundaries are also electrically insulating with the poloidal magnetic field mapped to external potential fields above the outer boundary and below the inner boundary. An insulating inner boundary condition is unrealistic for a model of the Earth's inner core boundary; however, given the simplicity of this intermediate dynamo model, we chose to keep the model simple and use this convenient boundary condition.

Equations (1)-(4) are solved in a manner similar to that described in GlatzMAIER (1984). The divergence-free velocity and magnetic field are written in terms of poloidal and toroidal 
scalar functions such that the mass and magnetic flux conservation equations (1) and (3) are automatically satisfied everywhere in space and time. These scalar functions and the pressure and density perturbations are expanded in axisymmetric spherical harmonics to resolve their latitudinal structure and in Chebyshev polynomials to resolve their radial structure. The radial component of the momentum equation (2), the divergence of the horizontal component of the momentum equation, and the radial component of the curl of the momentum equation provide a coupled system of differential equations (in radius) for the spherical harmonic coefficients of the poloidal and toroidal velocity fields and the pressure perturbation. The radial component of the magnetic induction equation (4) and the radial component of the curl of the magnetic induction equation provide differential equations (in radius) for the spherical harmonic coefficients of the poloidal and toroidal magnetic fields. The nonlinear Lorentz force and magnetic induction term are treated explicitly and calculated via a spectral transform method; the linear terms are treated implicitly. These equations are solved with a Chebyshev collocation method each timestep.

Once the magnetic field is updated for a given timestep, the velocity and pressure need to be updated. Since we wish to study solutions with a small Ekman number and an Elsasser number close to unity, i.e., Coriolis forces large compared with viscous forces and nearly in balance with the Lorentz forces, the Coriolis force needs to be treated very carefully to obtain an accurate velocity. We investigate two different numerical methods that are designed with this in mind. The first neglects the inertial term, which is assumed small, in Eq. (2) and, instead of having a buoyancy term in Eq. (2), prescribes a time-independent thermal wind that is added to the velocity perturbation solution each timestep. It is this total velocity field that advects the magnetic field in the magnetic induction equation (4). The momentum equation (2) is now a diagnostic equation for the velocity and pressure perturbations given the updated Lorentz force. The spherical harmonic representation of the Coriolis force couples the degree $l$ of the poloidal velocity and pressure with degrees $l \pm 1$ of the toroidal velocity. Therefore, instead of treating the Coriolis term explicitly as is done in GlatzMAIER (1984), we solve a sparse block banded matrix equation of order $3 L$ ( $L$ is the largest spherical harmonic degree of the truncated expansion) with each block being a dense matrix of order $N$ (the largest degree Chebyshev polynomial). The vector solution is a combination of the poloidal and toroidal velocity fields and the pressure perturbation, with a total order of $3 L \times N$. The numerical difficulty with this system is that the dominant Coriolis terms occupy off-diagonal blocks. We solve the system each timestep using a direct banded matrix solver, with an extra iteration step on the error vector to reduce its magnitude.

The second numerical method retains the inertial term in Eq. (2) and prescribes a timeindependent normalized density field for the buoyancy term. Now, in order to maintain numerical stability, the timestep is limited by additional Courant constraints due to inertial oscillations and the Alfven velocity (ST. PIERRE, 1993). In order to satisfy the inertial oscillation constraint, we subcycle on the linear terms in the momentum equation with a timestep less than $(2 \Omega)^{-1}$ using a Lorentz force linearly interpolated in time between the larger timesteps that are used to integrate the magnetic induction equation. To treat the Coriolis term accurately, we iterate during each subcycle timestep using a block Jacobi method until the maximum relative change is less than $10^{-5}$. The larger magnetic timestep must satisfy the Courant conditions based on the fluid and Alfven velocities for a given numerical mesh which are assumed to be less constraining than the inertial oscillation constraint. Therefore, instead of solving one large coupled system of equations with a direct matrix solver each timestep, as is done in the first method, we solve many small matrices each timestep by computing the Coriolis terms with the velocities from the previous iteration step. That is, each spherical harmonic degree of the radial component of the curl of the momentum equation can be solved separately for its contribution to the toroidal velocity. Likewise, each spherical harmonic degree of the poloidal velocity and pressure perturbation is obtained from the radial component of the momentum equation and the divergence of 
the horizontal component of the momentum equation which are still coupled and therefore solved simultaneously.

After updating the velocity and pressure by one of these two methods, we have completed the solution for this timestep and can start the next by returning to the induction equation to compute a new $\mathbf{B}$ and Lorentz force, as described earlier. The advantage of the first method is that larger timesteps can be used that are only limited by the normal Courant condition due to the fluid velocity which could be much smaller than the Alfven velocity. However, we found for our solutions that the second method requires almost an order of magnitude less computer memory and almost an order of magnitude less computer time than the first method if the large timestep in the second method is not many times larger than the inertial oscillation constraint. For cases that have relatively small inertial terms, the two methods obtain essentially identical solutions when the prescribed density perturbations in the second method produce a thermal wind like that prescribed in the first method.

For all the solutions presented here, we set the ratio of the inner to outer radii, $r_{i} / r_{0}$, to 0.35, Prandtl number, $\nu_{0} / \eta_{0}$, to unity, and the Ekman number, $\nu_{0} /\left(\Omega d^{2}\right)$, to $2.7 \times 10^{-6}$, where $d=r_{0}-r_{i}$. The spatial resolution is 65 Chebyshev radial levels and 64 Legendre latitudinal levels. In spherical harmonic space, this represents all degrees up to 42 . Each solution has been integrated for about 50,000 steps with a timestep of about $10^{-4} d^{2} / \eta_{0}$. The Reynolds number, $V_{\max } d / \nu_{0}$, for these solutions is about 1000 ; and the Elsasser number, $B_{\max }^{2} /\left(8 \pi \Omega \rho_{0} \eta_{0}\right)$, ranges from about 10 to 100 . We present three different cases driven by three different sets of alpha and omega fields.

\section{Results}

\subsection{Case one}

The first case was obtained by the first numerical method described above and with the following simple prescriptions for the alpha and omega fields (RoBERTs, 1972).

$$
\begin{gathered}
\alpha=\left(\frac{R_{\alpha} \eta_{0}}{d}\right) \cos \theta, \\
\omega=\left(\frac{R_{\omega} \eta_{0}}{d^{3}}\right) r .
\end{gathered}
$$

Plots of these fields are displayed in Fig. 1a. Alpha represents minus the helicity $(\mathbf{v} \cdot \nabla \times \mathbf{v})$ times a correlation time. Here alpha is assumed positive in the northern hemisphere and negative in the southern hemisphere. The thermal wind is assumed to be a retrograde wind (westward relative to the eastward rotating frame of reference) with a magnitude that increases with radius. $R_{\alpha}$ and $R_{\omega}$ are the alpha and omega Reynolds numbers, $\theta$ is colatitude, and the prescribed thermal wind is $\omega r \sin \theta$. We choose $R_{\alpha}=1$ and $R_{\omega}=-3000$ (BARENGHI and JonES, 1991). The dynamo number, defined as $D=R_{\alpha} R_{\omega}$, is then -3000 , about 2.18 times the critical dynamo number needed to generate a solution from small perturbations (BARENGHI and JONES, 1991). Note that the critical dynamo number is relatively insensitive to the ratio $r_{i} / r_{0}$ (ROBERTS, 1972).

Although our numerical model is somewhat different than the magnetostrophic models of Roberts (1972) and BARENGHI and JONES (1991), our periodic solution, characterized by a dynamo wave continually propagating toward the equator in both hemispheres, is very similar to their solutions. In a series of snapshots, Fig. 1b illustrates the dipole family type solution (toroidal magnetic field is anti-symmetric with respect to the equatorial plane) and the propagating phase of the magnetic field. As noted above, this symmetry is not imposed by the model but, instead, a result of the simply defined $\alpha$ and $\omega$ fields (5,6). As proposed by PARKER (1955), helical fluid motions twist toroidal magnetic field producing poloidal magnetic field (the "alpha effect") and 
differential rotation shears poloidal magnetic field producing toroidal magnetic field (the "omega effect"). The particular alpha and omega fields that have been prescribed cause the poloidal and toroidal fields to continually be enhanced on the equatorward sides of their local maxima and be destroyed on the poleward sides. This, together with magnetic diffusion, causes the phase of the magnetic field profile to propagate toward the equatorial plane. Half of a magnetic cycle $\left(0.27 d^{2} / \eta_{0}\right)$ is depicted in Fig. 1b. Although the magnetic phase pattern and propagation is very similar to that obtained by ROBERTS (1972) and BARENGHI and JoNES (1991), our period is about four times larger than theirs. This discrepancy may be due to our fluid viscosity or our meridional circulation, neither of which are included in the other models.

The velocity field is also calculated, except for the thermal wind part of the toroidal velocity. The profiles of the toroidal velocity (differential rotation) and the poloidal velocity (meridional circulation) are relatively time independent. Typical profiles of the velocity fields are illustrated in Fig. 1c. Two meridional cells exist in each hemisphere and a retrograde toroidal flow peaks near the outer boundary in the equatorial plane.

Compared to the average toroidal kinetic energy, the average toroidal magnetic energy is $2 \times 10^{-1}$, the average poloidal magnetic energy is $5 \times 10^{-4}$, and the average poloidal kinetic energy is $4 \times 10^{-6}$. These energies all oscillate sinusoidally with a period of $0.27 d^{2} / \eta_{0}$, which is half the period required for the magnetic field to return to the same polarity configuration. They are all nearly in phase; however, the poloidal kinetic energy slightly leads the toroidal kinetic energy which slightly leads the toroidal magnetic energy which slightly leads the poloidal magnetic energy.

\subsection{Case two}

Our second case was also obtained by the first numerical method described above but uses the following prescriptions for the alpha and omega fields introduced by BRAGINSKY and ROBERTS (1987) and applied here for $r_{i} \geq r \geq r_{0}$.

$$
\begin{gathered}
\alpha=0 \quad \text { for } \quad s \leq 0.8 r_{0}, \\
\alpha=\left(\frac{20 R_{\alpha} \eta_{0}}{r_{0}^{2}}\right) z\left(1-\left(\frac{z}{z_{1}}\right)^{6}\right) \sin \left(\pi\left(9-\frac{10 s}{r_{0}}\right)\right) \quad \text { for } \quad 0.8 r_{0}<s \leq r_{0}, \\
\omega=-\left(\frac{3 R_{\omega} \eta_{0}}{r^{4}}\right) s^{2}\left(1-\left(\frac{r}{r_{0}}\right)^{2}\right) .
\end{gathered}
$$

Here $s=r \sin \theta, z=r \cos \theta$, and $z_{1}=r_{0}\left(1-\left(s / r_{0}\right)^{2}\right)^{1 / 2}$. These fields are plotted in Fig. 2a. The alpha field now has both signs in each hemisphere and is nonzero only near the outer boundary at low latitude. The thermal wind is retrograde as in case one; but now it has a peak westward velocity near mid-depth in the equatorial plane.

With the above definitions (7)-(8), the critical dynamo number is approximately 5120 (P. W. Hofflin and R. W. James, these proceedings). We have generated three different solutions for this case: a subcritical steady state solution with $R_{\alpha}=10$ and $R_{\omega}=500$, a supercritical steady state solution with $R_{\alpha}=3$ and $R_{\omega}=3000$, and a supercritical periodic solution $R_{\alpha}=10$ and $R_{\omega}=3000$. Again, although our numerical model is different than the magnetostrophic model of BRAGINSKY (1978) and ROBERTS (1989), including the existence of an inner core boundary in our model, our steady state solutions outside the inner core tangent cylinder are quite similar to theirs. Figure $2 \mathrm{~b}$ shows the magnetic fields for our steady state subcritical and supercritical solutions. It is interesting that this $\alpha \omega$ dynamo model is steady at criticality, unlike case one. PARKER (1979) suggests that steady dynamo solutions are preferred when the sources are localized, as is the $\alpha$-effect in this case (Fig. 2a). 


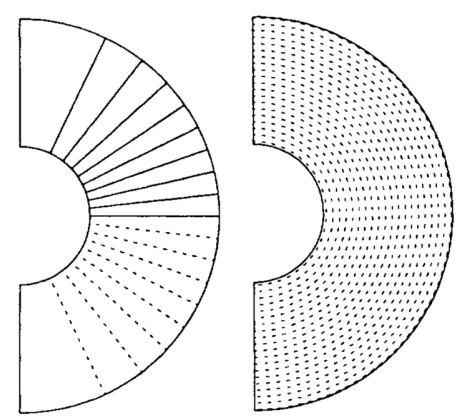

Fig. 1a. Prescribed alpha (on the left) and omega (on the right) fields for case 1 plotted in radius and latitude. Solid (broken) contours represent positive (negative) values. The omega field becomes more negative with increasing radius.
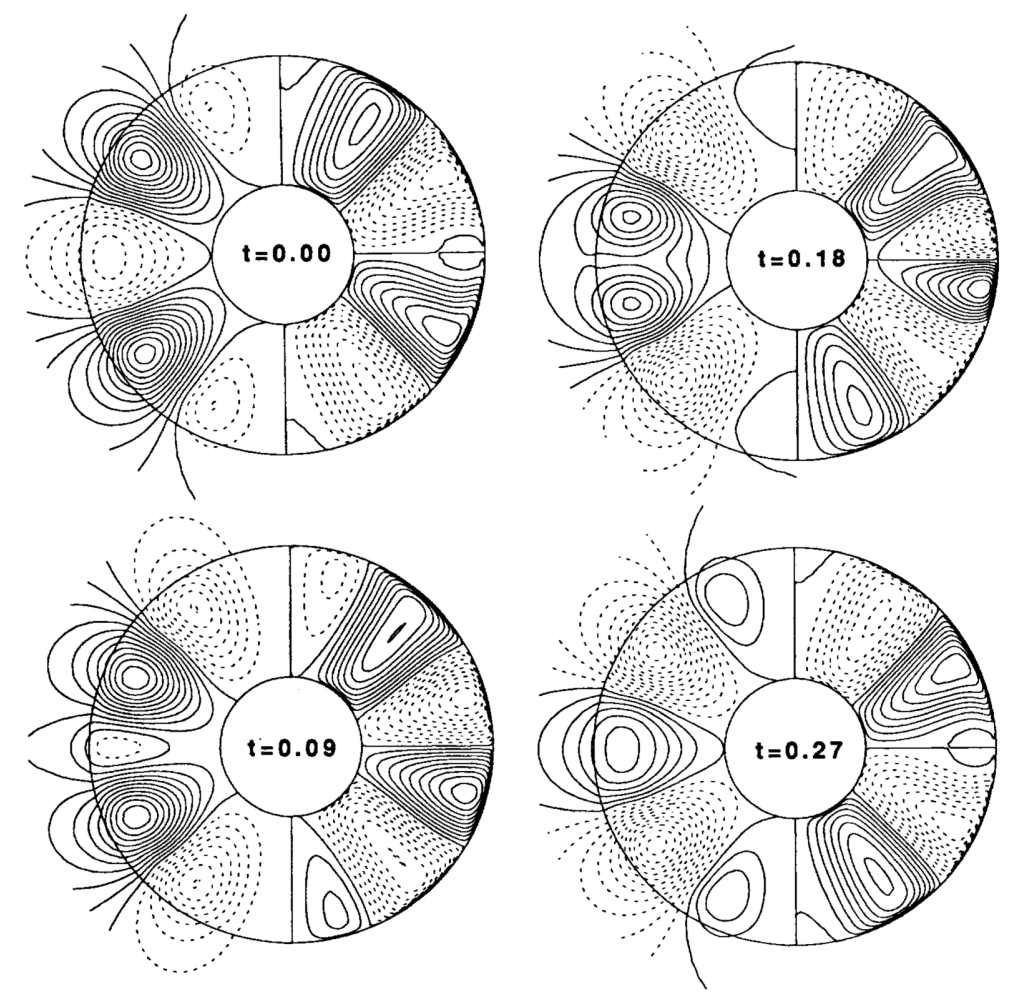

Fig. 1b. A series of snapshots of the axisymmetric magnetic field solution for case 1. The times are scaled by $d^{2} / \eta_{0}$. Each plot shows field lines of the poloidal field on the left and contours of the toroidal field on the right. Solid (broken) field lines represent clockwise (counter-clockwise) directed poloidal field. The potential field in the inner core is not plotted. Solid (broken) contours represent eastward (westward) directed toroidal field. 


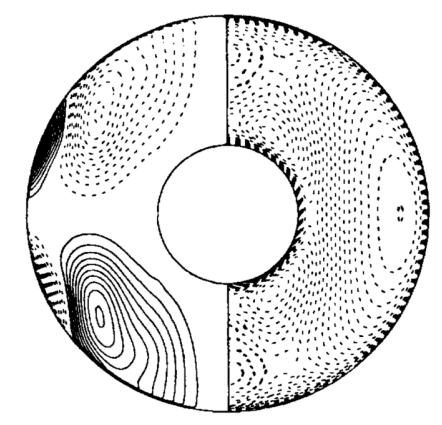

Fig. 1c. A snapshot of the axisymmetric velocity solution for case 1 . Stream lines of the poloidal velocity are displayed on the left and contours of the toroidal velocity are displayed on the right. Solid (broken) stream lines represent clockwise (counter-clockwise) directed fluid circulation. Solid (broken) contours represent eastward (westward) directed fluid flow.

When we increased the dynamo number to $3 \times 10^{4}$, we obtained a periodic (although not sinusoidal) solution. Half of a magnetic cycle $\left(0.24 d^{2} / \eta_{0}\right)$ is illustrated in a series of snapshots in Fig. 2c. The symmetry that is preferred (but not forced) in the lower dynamo number steady state solutions (Fig. 2b) is no longer preferred at the higher dynamo number (Fig. 2c). Instead, the peak magnetic field strength oscillates between the northern and southern hemispheres while maintaining its primary polarity in each hemisphere. That is, the magnetic field is mainly of the dipole family, but has an oscillating quadrupole component. While the toroidal magnetic field is peaking in one hemisphere, the same polarity attempts to invade the other hemisphere; but it never is quite able to overcome the primary polarity in that hemisphere.

A snapshot of the velocity field is shown in Fig. 2d. The toroidal velocity field is relatively time independent. It is retrograde with it's magnitude increasing with decreasing distance to the rotation axis. The poloidal velocity field pattern oscillates in a manner that is somewhat similar to that of the toroidal magnetic field. The main part of the meridional circulation is similar to the equatorially symmetric circulation that exists in our steady state solutions (not shown); but in addition there is a very time dependent circulation that crosses the equatorial plane (Fig. $2 \mathrm{~d}$ ).

Compared to the average toroidal magnetic energy, the average toroidal kinetic energy is $10^{-1}$, the average poloidal magnetic energy is $4 \times 10^{-2}$, and the average poloidal kinetic energy is $10^{-4}$. These energies oscillate with a period of $0.24 d^{2} / \eta_{0}$, which again is half the required period for the magnetic field to return to the same configuration.

\subsection{Case three}

Our third case was obtained by the second numerical method described above. The prescribed alpha and density perturbation are defined as

$$
\begin{gathered}
\alpha=-\left(\frac{R_{\alpha} \eta_{0}}{d}\right) F_{\alpha}(r, \theta), \\
\frac{\rho}{\rho_{0}}=R_{\omega} F_{\omega}(r, \theta),
\end{gathered}
$$

where $F_{\alpha}$ is the longitudinally averaged helicity (normalized to unity) and $F_{\omega}$ is the longitudinally averaged density perturbation (normalized to unity) of a non-magnetic three-dimensional rotating thermal convection simulation computed by GLATZMAIER and Olson (1993). This three-dimensional simulation had an Ekman number of $10^{-4}$ and a Rayleigh number of $6.67 \times 10^{6}$ (50 times critical for this Ekman number). Although this thermal convection solution is still 
far from representing convection in the Earth's liquid core, it provides more self-consistent mean field profiles for the alpha and density prescriptions. Plots of alpha and density perturbation are displayed in Fig. 3a. As in case 1, the alpha field is mainly positive (negative) in the northern (southern) hemisphere. As in case 2, it has both signs in both hemispheres; but here is nonzero nearly everywhere. The density perturbation is most negative near the inner boundary and in the equatorial plane. Notice in Fig. 3a how both fields are affected by the inner core tangent cylinder.

With the above definitions (9)-(10), we present a solution for $R_{\alpha}=113$ and $R_{\omega}=10^{-5}$ which is very time dependent and does not return to a previous configuration. Figure $3 \mathrm{~b}$ shows a series of snapshots separated by time increments of $0.02 d^{2} / \eta_{0}$. At times the magnetic field pattern is mainly of the dipole family and at other times it is mainly of the quadrupole family. As can be seen in Fig. 3b, the magnetic field patterns tend to propagate from the outer part of the shell toward the rotation axis and tend to diffuse away after passing through the inner core tangent cylinder.

A snapshot of the velocity profiles is shown in Fig. 3c. The toroidal velocity has a weak retrograde jet near the inner core in the equatorial plane and strong retrograde flow inside the inner core tangent cylinder. This retrograde zonal flow profile is qualitatively similar to that

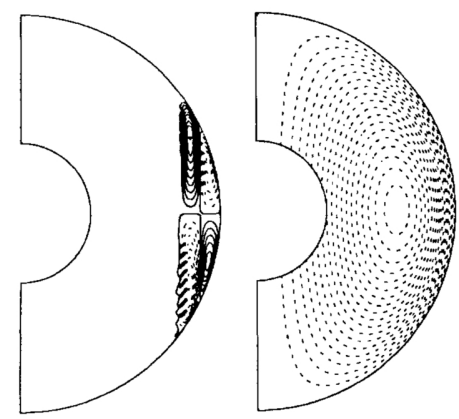

Fig. 2a. Plots of the prescribed alpha (on the left) and omega (on the right) fields for case 2 (as described in Fig. 1a). The omega field is most negative near mid-depth in the equatorial plane.
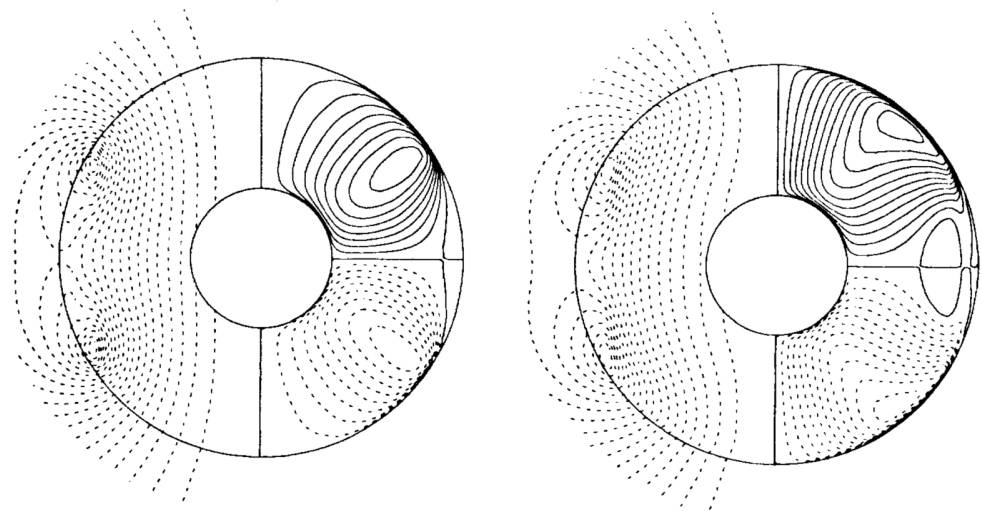

Fig. 2b. Plots of the steady state axisymmetric magnetic field solutions for case 2 (as described in Fig. 1b). The plot on the left is the subcritical solution $\left(R_{\alpha}=10, R_{\omega}=500\right)$; the plot on the right is the supercritical solution ( $R_{\alpha}=3, R_{\omega}=3000$ ). 

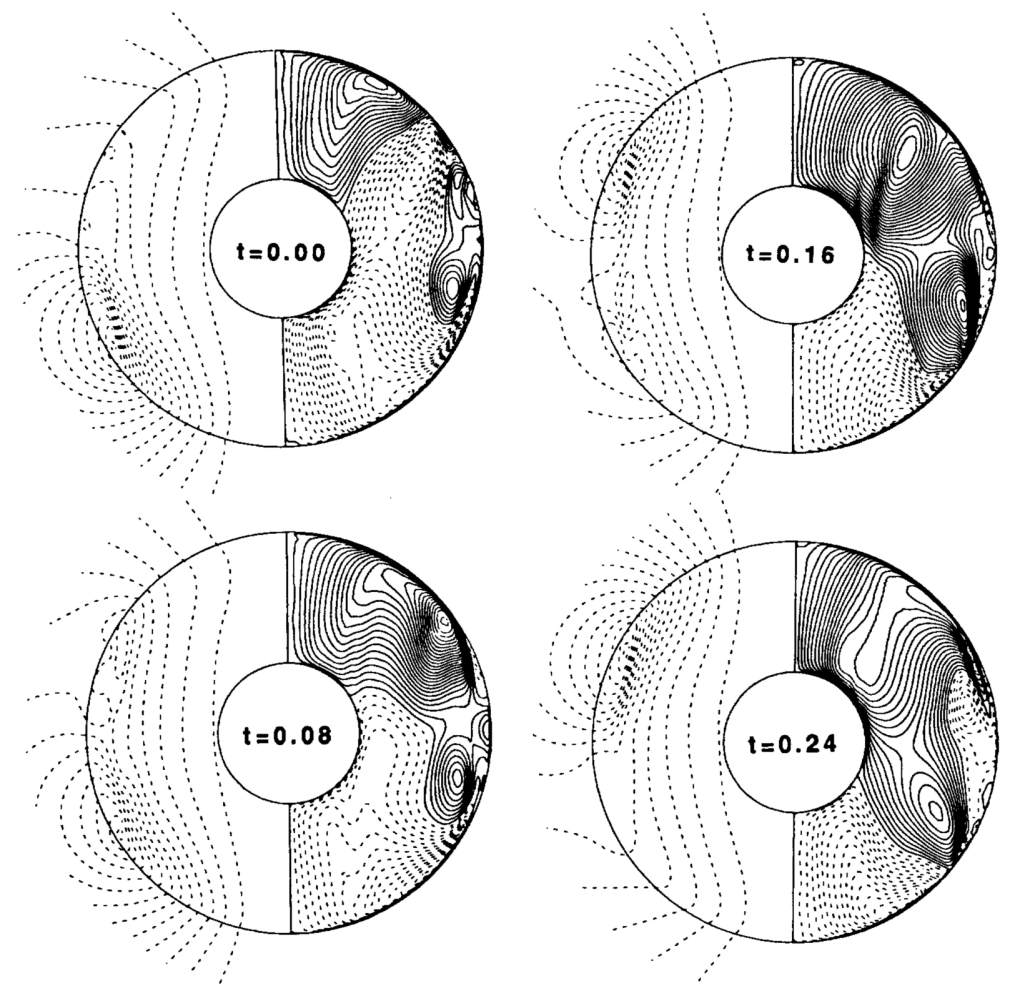

Fig. 2c. A series of snapshots (as described in Fig. 1b) of the time-dependent axisymmetric magnetic field solution for case $2\left(R_{\alpha}=10, R_{\omega}=3000\right)$.

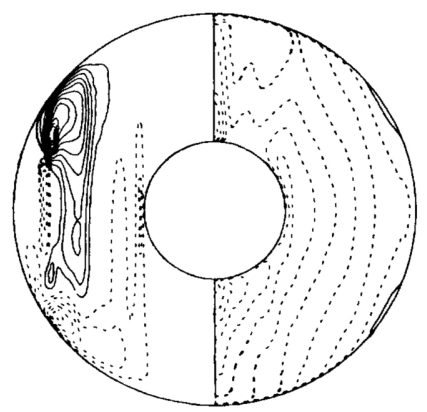

Fig. 2d. A snapshot of the time dependent velocity solution (as described in Fig. 1c) for case $2\left(R_{\alpha}=10\right.$, $R_{\omega}=3000$ ). 

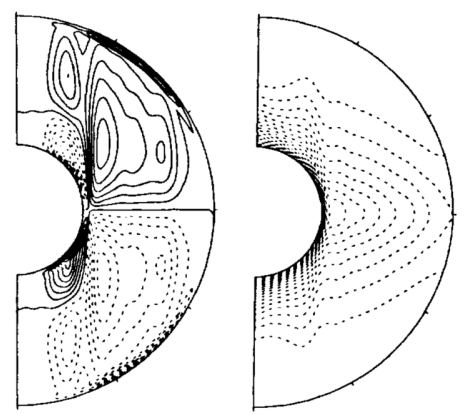

Fig. 3a. Plots of the prescribed alpha (on the left) and density perturbation (on the right) fields for case 3 (as described in Fig. 1a). The density perturbation is most negative near the inner boundary.
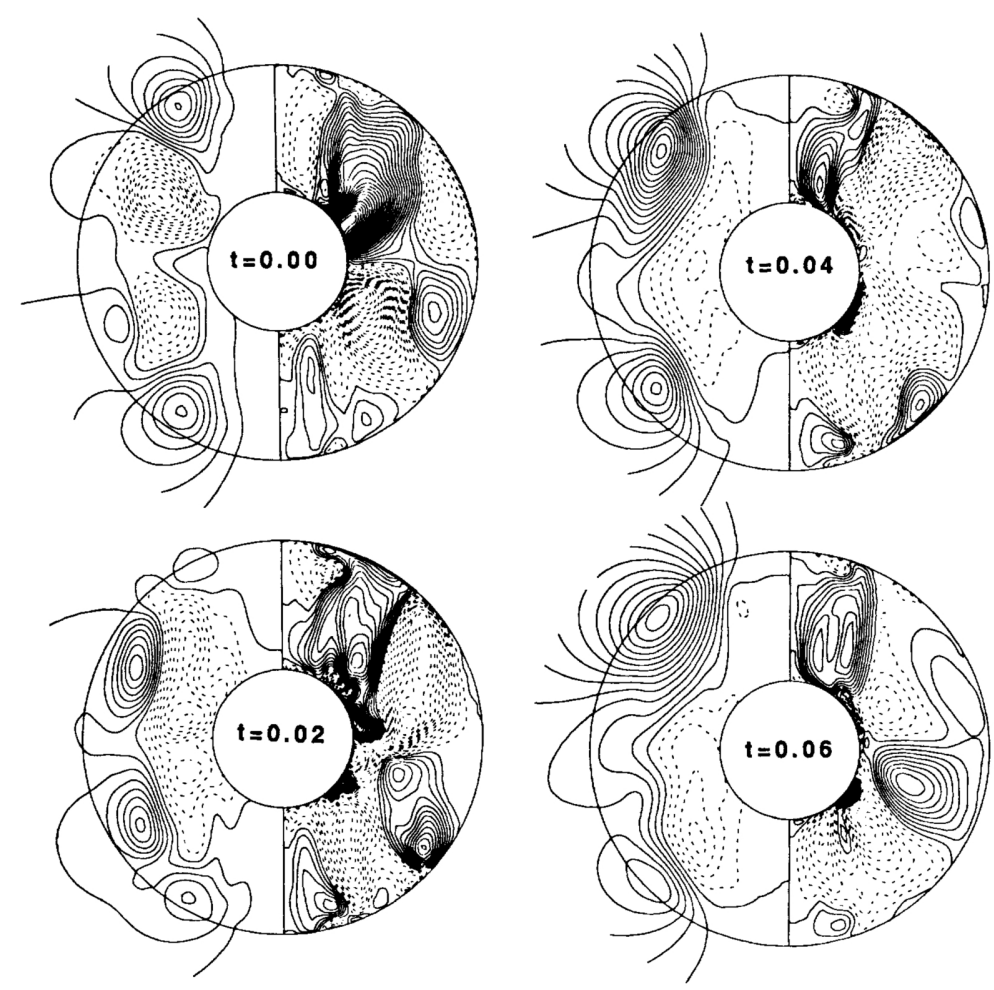

Fig. 3b. A series of snapshots (as described in Fig. 1b) of the axisymmetric magnetic field solution for case 3. 


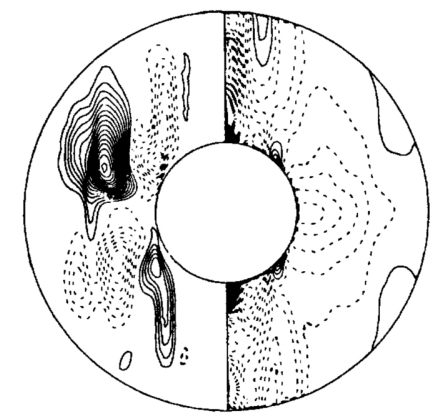

Fig. 3c. A snapshot of the velocity solution (as described in Fig. 1c) for case 3.

generated by the three dimensional thermal convection simulation of GLATZMAIER and OLSON (1993) from which the prescribed density field was obtained. While the toroidal velocity field changes little with time, the poloidal velocity is quite time dependent. The average kinetic energy is usually one to two orders of magnitude less than the average magnetic energy.

\section{Conclusions}

We have described two versions of an intermediate dynamo model that we have used to obtain three very different time-dependent axisymmetric magnetic and velocity field solutions. The first case is an equatorward propagating dynamo wave that has been studied in the past. In the second case, we obtained steady state solutions at low dynamo numbers, similar to what have been obtained in the past, and a new time dependent solution at a higher dynamo number that has the peak field strength oscillating between the northern and southern hemispheres. The third case is a new time dependent solution that is not periodic but is characterized by phase propagation toward the rotation axis. It is driven by profiles for the alpha and omega effects that were obtained from a three-dimensional thermal convection simulation.

Presumably many variations of these solutions could be obtained and analyzed by using different values of $R_{\alpha}$ and $R_{\omega}$. Likewise many different solutions could be obtained by using different profiles of the prescribed alpha and omega fields. However, it is our opinion that, if one wishes to investigate and study in detail the type of dynamos that exist in the interiors of planets, one needs a self-consistent three-dimensional dynamo model that is driven by, and feeds back onto, thermal and compositional convection and at most relies on prescribed diffusivities.

We would like to thank Y. Honkura and another referee for their helpful suggestions for writing this paper. This research was supported by the Los Alamos branch of the Institute for Geophysics and Planetary Physics, University of California and was performed under the auspices of the US Department of Energy.

\section{REFERENCES}

BARENGHI, C. F. and C. A. JonEs, Nonlinear planetary dynamos in a rotating spherical shell I. Numerical methods, Geophys. Astrophys. Fluid Dyn., 60, 211-243, 1991.

BrAGinSKY, S. I., Nearly axially symmetric model of the hydromagnetic dynamo of the Earth I, Geomagn. Aeron., 15, 122-128, 1975.

BRAGINSKY, S. I., Nearly axially symmetric model of the hydromagnetic dynamo of the Earth II, Geomagn. Aeron., 18, 225-231, 1978.

Braginsky, S. I. and P. H. Roberts, A model-Z geodynamo, Geophys. Astrophys. Fluid Dyn., 38, 327-349, 1987. 
FEARN, D. R. and M. R. E. Proctor, On the computation of steady self-consistent spherical dynamos, Geophys. Astrophys. Fluid Dyn., 38, 293-325, 1987.

Gilman, P. A., Dynamically consistent nonlinear dynamos driven by convection in a rotating spherical shell. II. Dynamos with cycles and strong feedbacks, Astrophys. J. Suppl., 53, 243-268, 1983.

Gilman, P. A. and J. Miller, Dynamically consistent nonlinear dynamos driven by convection in a rotating spherical shell, Astrophys. J. Suppl., 46, 211-238, 1981.

Glatzmaier, G. A., Numerical simulations of stellar convective dynamos. I. The model and method, J. Comp. Phys., 55, 461-484, 1984.

Glatzmaier, G. A., Numerical simulations of stellar convective dynamos. II. Field propagation in the convection zone, Astrophys. J., 291, 300-307, 1985a.

Glatzmaier, G. A., Numerical simulations of stellar convective dynamos. III. At the base of the convection zone, Geophys. Astrophys. Fluid Dyn., 31, 137-150, 1985b.

Glatzmaier, G. A. and P. Olson, Highly supercritical thermal convection in a rotating sprical shell: Centrifugal vs. radial gravity, Geophys. Astrophys. Fluid Dyn., 70, 113-136, 1993.

Hollerbach, R., A direct spectral solution of the Ekman and Stewartson layers in a rotating spherical shell, $J$. Comp. Phys., 1992 (submitted).

Olson, P., Toroidal flow in the outer core and the thermal regime below the core-mantle boundary, Geophys. Res. Lett., 16, 613-616, 1989.

PARKer, E. N., Hydromagnetic dynamo models, Ap. J., 122, 293-314, 1955.

PARker, E. N., Cosmical Magnetic Fields: Their Origin and Their Activity, Clarendon Press, Oxford, 1979.

Roberts, P. H., Kinematic dynamo models, Philos. Trans. R. Soc., A272, 663-698, 1972.

Roberts, P. H., From Taylor state to model-Z, Geophys. Astrophys. Fluid Dyn., 49, 143--160, 1989.

Roberts, P. H. and A. M. Soward, Dynamo theory, Ann. Rev. Fluid Mech., 24, 459-512, 1992.

St. PIerre, M. G., The stability of the magnetostrophic approximation I: Taylor state solutions, 1993 (submitted).

ZhANG, K.-K. and F. H. Busse, Convection driven magnetohydrodynamic dynamos in rotating spherical shells, Geophys. Astrophys. Fluid Dyn., 49, 97-116, 1989.

ZHANG, K.-K. and F. H. Bisse, Generation of magnetic fields by convection in a rotating spherical shell of infinite Prandtl number, Phys. Earth Planet. Int., 59, 208-222, 1990. 